\title{
Clinical practice guideline for image-guided multimode tumour ablation therapy in hepatic malignant tumours
}

\author{
G.Z. Wang $M D P h D,{ }^{*}$ X.H. He $M D P h D,{ }^{*} Y$. Wang $M D,{ }^{*}$ L.C. Xu MD, * H.Z. Huang $M D, *$ Y.H. Wang $M D,{ }^{*}$ \\ Z. Shen MD PhD, ${ }^{\dagger}$ X.D. Qu MD PhD, ${ }^{\ddagger}$ X.Y. Ding MD PhD, ${ }^{\S}$ J.J. Yang MD PhD,
}

\begin{abstract}
Multimode tumour ablation therapy is a treatment method that combines cryoablation with radiofrequency ablation, guided by medical imaging technology and based on precise planning, targeting, monitoring, and control of the thermal energy delivered, with the aim of achieving a whole-body antitumour immune response to malignant tumours. To develop standardized criteria for the application of multimode tumour ablation therapy to malignant hepatic tumours, to facilitate actualization of the criteria in various hospitals, and to ensure therapeutic efficacy and safety, the Society of Interventional Therapy of the Chinese Anti-Cancer Association and the Solid Tumor Theranostics Committee of the Shanghai Anti-Cancer Association assembled experts who specialize in oncology to discuss this treatment method and to arrive at a clinical practice consensus guideline for the indications, contraindications, and techniques of multimode tumour ablation therapy for malignant hepatic tumours.
\end{abstract}

Key Words Cryosurgery, cryoablation, radiofrequency ablation, liver neoplasms, consensus statements

Curr Oncol. 2019 October;26(5):e658-e664

www.current-oncology.com

\section{INTRODUCTION}

With the rapid development of medical imaging techniques - ultrasonography, computed tomography (ст), magnetic resonance imaging (MRI) - since the early 2000s, image-guided tumour ablation, being an effective, repeatable, low-cost, minimally invasive, and safe treatment, developed tremendously and became a well-established approach for local cancer therapy. Percutaneous tumour ablation by physical or chemical means is performed under the guidance of medical imaging, causing tumour necrosis and loss of activity, with the purpose of controlling and eliminating the tumour ${ }^{1,2}$. Clinically, the most commonly used ablation therapies include thermal ablation [for example, radiofrequency ablation (RFA), microwave ablation, high-intensity focused ultrasound ablation, laser ablation], cryoablation (argon-helium cryoablation and liquid nitrogen cryosurgery), and chemical ablation by local injection of anhydrous ethanol. In recent years, irreversible electroporation has been gradually applied in clinical practice ${ }^{1,3,4}$. The extensive development of image-guided tumour ablation has increased the number of potential applications for the technique in recurrence and metastasis of malignant tumours and has changed the concept of traditional surgical treatment. More and more studies report that the immune response or abscopal effect can be induced by local ablation ${ }^{5-9}$.

The recently developed technique of multimode ablation therapy combining cryoablation with RFA in one procedure can potentially elicit an antitumour immune response $\mathrm{e}^{10-13}$. Based on integrating multiple imaging techniques with an intelligent navigation system, multimode ablation therapy can precisely control the ablation margin and zone by modifying the energy or thermal power and temperature field, providing an effective clinical solution for the treatment of hepatic malignant tumours ${ }^{2}$.

To attain greater worldwide adoption of multimode ablation therapy for hepatic malignant tumours and to ensure safety and clinical efficacy, the Society of Interventional Therapy of the Chinese Anti-Cancer Association and the Solid Tumor Theranostics Committee of the Shanghai Anti-Cancer Association arranged for a multidisciplinary

Correspondence to: Wen-Tao Li or Guang-Zhi Wang, Department of Interventional Oncology, Fudan University Shanghai Cancer Center, 270 Dong'an Road, Xuhui, Shanghai 200032 P.R.C.

E-mail: liwentao98@126.com or guangzhiwang2000@163.com — DOI: https://doi.org/10.3747/co.26.5423 
group of oncology experts to discuss the issues and to arrive at a consensus clinical practice guideline about the indications, contraindications, and techniques of multimode tumour ablation therapy for malignant liver tumours.

\section{Principles of Multimode Tumour Ablation Therapy}

In multimode tumour ablation therapy, ultralowtemperature cryoablation causes irreversible frostbite to tumour cells and kills tumour tissue at temperatures ranging from $-40^{\circ} \mathrm{C}$ to $-180^{\circ} \mathrm{C}$. When cryoablation takes tissue temperature below $-40^{\circ} \mathrm{C}$, cells are destroyed through ice crystal formation and osmotic shock. At the same time, cell metabolism is disrupted. With a further reduction in temperature, ice crystals begin to form outside the cells, resulting in extracellular hyperosmolarity that leads to extravasation of intracellular fluid and cell dehydration. During thawing, the osmotic gradient reverses, and the influx of extracellular fluid leads to cell swelling and membrane rupture ${ }^{14,15}$. The subsequent application of thermal ablation causes acute coagulative necrosis of tumour tissue. At high temperatures, protein denaturation and cell membrane melting occur, and the cells die instantly ${ }^{16}$.

The goal of the multimode ablation technique is to disintegrate tumour cells in situ by sequentially implementing rapid temperature changes in tumour tissue, including alternating the temperature field change, achieving a corresponding thermal stress and mechanical force from the interruption and reperfusion of blood flow in the tissue. Multimode ablation completely kills tumour cells and can lead to the release of maximal amounts of tumour antigen, which induces active antitumour immunity ${ }^{10}$. Multimode ablation is not a simple superposition of cryoablation and thermal ablation: its effectiveness for the treatment of tumours by multiple modes (freezing, heating, and immune response) relies on precise regulation of thermal power and temperature field ${ }^{11-13,17,18}$.

Multimode ablation therapy is therefore defined as a novel image-guided tumour ablation treatment combining cryoablation and RFA, with planning that integrates CT, MRI, ultrasonography, electromagnetic navigation, and other morphologic and functional imaging devices, targeted by a precise spatial positioning system and controlled by precise regulation of the thermal dose and the temperature field ${ }^{19,20}$. During the alternation of freezing and RFA, the quantitative planning of precise temperature control and the ablation process in focal areas might not only improve the homogeneity and efficiency of heating, but also potentially destroy tumour cells and their circulatory system in situ. Meanwhile, maximal amounts of active tumour antigen can be released, achieving the goals of local treatment, stimulation of systemic immunity, and control of tumour recurrence and metastasis ${ }^{18,20}$.

\section{PRACTICE GUIDELINE}

\section{Platform}

Precise positioning and real-time navigation by image guidance depend on the multimode imaging platform. The pathway design and tumour targeting are based on 3-dimensional reconstruction and fusion of a variety of medical imaging data (CT, MRI, and ultrasonography) on a PACs (picture archiving and communication system) image-processing workstation. All pre-procedural strategy planning, intraprocedural monitoring, and post-procedural evaluation are conducted as a single treatment procedure ${ }^{19,21}$. Using quantitative navigation technologies, cryoablation and RFA are sequentially performed to achieve multimode ablation.

\section{Indications and Contraindications}

In patients with hepatic malignant tumours, multimode ablation could be suitable for

those who are not candidates for curative surgical resection because of cardiorespiratory comorbidity, insufficient vital lung function, or older age; those who refuse to accept surgical resection; or those who have a tumour with a maximal diameter of $5 \mathrm{~cm}$ or less, or in the presence of multiple tumours, who have 5 or fewer lesions ${ }^{2,22-25}$.

\section{Contraindications}

Because of the excellent tolerance for percutaneous local ablation, identifying an absolute contraindication for liver thermal ablation is difficult; the exceptions are patients with untreatable coagulopathies ${ }^{26}$. These are the contraindications for multimode ablation of hepatic tumours ${ }^{23,25,27}$ :

Child-Pugh class C liver function

Tumour invading the adjacent viscera

Severe dysfunction of liver, kidney, heart, lung, or brain; severe anemia or dehydration; and severe disorders of the nutrition metabolism that cannot be corrected or improved in the short term

Severe systemic infection or high fever $\left(>38.5^{\circ} \mathrm{C}\right)$

Poorly controlled malignant ascites or dyscrasia

Poorly controlled infection or active inflammation around the lesions and puncture-site infection or ulceration

- Tendency to severe hemorrhage, with a platelet count of $50 \times 10^{9} /$ L or less and severe coagulation dysfunction (anticoagulation therapy or antiplatelet agents should be withdrawn for at least 5-7 days before ablation)

- Extensive extrahepatic metastases, with an expected survival of less than 3 months

- An Eastern Cooperative Oncology Group performance status of 3 or greater

Implanted pacemaker

\section{Procedure Preparation}

\section{Patient Assessment and Imaging}

Patients should be evaluated with a careful review of their medical history, a physical examination, and a review of recent imaging data. Selection of the multimode procedure should be a multidisciplinary decision, with the associated discussion recorded.

\section{Imaging examination}

Within 2 weeks before the procedure, contrastenhanced ultrasonography, enhanced CT, or enhanced MRI of liver; chest radiography; and integrated 
positron-emission tomography-CT to exclude or detect distant metastases, if applicable, should be performed.

\section{Cardiac function test}

Electrocardiography, echocardiography (optional for elderly patients), and any other required test should be obtained.

\section{Laboratory tests}

Within 2 weeks before the procedure, routine blood, urine, and stool tests; coagulation; liver and kidney function; blood glucose, tumour markers, type, and infection tests should be performed.

\section{Pathology examination}

Percutaneous biopsy of the tumour should be performed as confirmation of the diagnosis before the procedure.

\section{Equipment and instruments}

Image-guidance equipment, RFA therapeutic apparatus, cryoablation apparatus, cryoablation antennas, radiofrequency antennas, electric defibrillator, and monitoring equipment should be present and in proper working order.

\section{Drug preparation}

Anesthetics, sedatives, analgesics, antiemetics, hemostatic drugs, rescue medicines, and other conventional drugs should be prepared before the procedure.

\section{Patient preparation}

- Informed consent should be obtained.

- Any anticoagulant and blood-activating drug should be discontinued more than 1 week before the procedure.

- Before local anesthesia, fasting from solid and liquid foods for 4 hours is required; before general anesthesia, fasting from solid foods for 12 hours and from liquids, including water, for 4 hours is required.

- The skin surface should be prepared for the procedure, if necessary.

- Before the procedure, intravenous access should be established ${ }^{2,22-25}$.

\section{Anesthetic Method}

Anesthesiologists should conduct a pre-anesthetic assessment per the classification criteria of the American Society of Anesthesiologists ${ }^{28}$. Local anesthesia, intravenous analgesia, intravenous anesthesia, epidural anesthesia, or tracheal anesthesia for punctures is selected according to the patient's condition. General anesthesia or local anesthesia can be applied for the procedure.

\section{Ablation Procedure}

Once the multimode ablation technique is selected, imaging modalities-CT, ultrasonography, positron-emission tomography, MRI-are used during the procedure. The most commonly applied and accurate image-guided technique is ст. Multimode ablation includes planning, targeting, ablation, monitoring, intraprocedural modifications, and assessment of treatment response.

\section{Planning}

Based on pre-procedural imaging, the tumour, including location, size, shape, and relationship with adjacent organs, is targeted; the appropriate body position for the patient is selected; and the puncture site and path to the lesion are determined. The puncture path must avoid large blood vessels, the bile duct, and other important organs.

\section{Targeting}

After anesthesia, the ablation antenna is inserted along the puncture path that was determined during preprocedural planning. The ст imaging is confirmed and the 3-dimensional reconstructed image is used to observe whether the ablation applicator has been inserted into the target lesion.

\section{Multimode Ablation Method}

Multimode ablation is achieved using cryoablation and RFA systems, with these treatment steps:

Based on the size and shape of the tumour, the number of cryoablation antennas and their puncture pathways are determined. The antennas are inserted into the tumour tissue under image guidance.

The cryoablation system is turned on, the scope of the ice ball is monitored, and the distance between the boundary of the frozen ball and the $5 \mathrm{~mm}$ margin of the tumour is maintained by adjusting the output power. Freezing lasts 5 minutes. After the cryoablation needle is retracted, rewarming occurs for 10-15 minutes.

Based on the size and shape of the tumour, the RFA needle is inserted into the tumour tissue under imaging guidance.

The RFA device is connected and ablation is performed in accordance with the predetermined power and time. The ablation area, including the $5 \mathrm{~mm}$ safety margin around the tumour, is maintained, and the ablation power and time are increased, if necessary, based on image guidance ${ }^{12}$.

\section{Temperature Field Control and Monitoring}

During treatment, the ablation power and time should be controlled based on the temperature field calculation ${ }^{1,3,4}$, the morphology of the ice ball, and the monitored coverage of the ablation area. The ablation area should be at least $5 \mathrm{~mm}$ beyond the tumour margin.

\section{Intraprocedural Modification}

If the actual ablation range does not achieve the expected effect because of vascular distribution or tissue characteristics, modification of the ablation treatment will be needed based on the information obtained from image guidance. Intraprocedural modification might simply require repositioning of an applicator and adjustment to the ablation parameters based on the imaging findings, or it might be as sophisticated as using an automated system that automatically terminates the ablation at a critical point during the procedure. 
Assessment of Immediate Treatment Response

After ablation, CT imaging or MRI should be performed to obtain a preliminary evaluation of the ablation effect and to identify complications such as pneumothorax or hemorrhage, if any.

\section{Complications and Side Effects}

The complications of multimode tumour ablation therapy are stratified into minor (A or B) or major (C-F) grades per the U.S. Society of Interventional Radiology criteria:

A. No therapy, no consequences

B. Nominal therapy, no consequences (includes overnight admission for observation only)

C. Requires therapy, minor hospitalization $(<48$ hours)

D. Requires major therapy, unplanned increase in level of care, prolonged hospitalization ( $>48$ hours)

E. Permanent adverse sequelae

F. Death

Based on the time of occurrence, complications can be divided into acute ( $<24$ hours), periprocedural ( 24 hours to 30 days), and delayed (>30 days) categories ${ }^{26}$. Sufficient pre-procedural preparation, standardized operative procedures, precise positioning, and minimal puncture time are important for reducing the incidence of complications.

\section{Pain}

Intraprocedural pain that is mild to moderate can be relieved with nonsteroidal anti-inflammatory drugs. Ample analgesics (such as opioids or sedatives) should be given for post-procedural pain that is moderate or severe, after exclusion of hemorrhage.

\section{Post-Ablation Syndrome}

The main manifestations of post-ablation syndrome are a low-grade fever, fatigue, general malaise, and nausea and vomiting after ablation, generally lasting 2-7 days. For patients with a greater ablated volume, the manifestations might last 2-3 weeks. Intensive care, infusion of fluids, pain relief, symptomatic treatment, and regular observation of hepatic and renal function should be carried out after ablation.

\section{Biliary-Cardiac Reflex}

Puncture or thermal stimulation of the biliary tract during the ablation can cause vagal excitation, slowing the heart rate and lowering blood pressure. Severe instances can lead to myocardial ischemia, arrhythmia, and even sudden cardiac arrest. Treatment should be ceased immediately, sedation and analgesia should be strengthened, and urgent treatment should be given if necessary.

\section{Intrahepatic Hematoma and Subcapsular Hepatic or Intra-abdominal Hemorrhage}

During the puncture step, rupture of the hepatic capsule or parenchyma of the tumour, vascular injury, and inadequate ablation of the needle tract can cause hemorrhage. Small hemorrhages can be treated conservatively.
Arterial embolization or ablation for hemostasis should be quickly performed if active arterial or other massive hemorrhage occurs. Active anti-shock treatment should be given for hemorrhagic shock, and surgery should be performed, if necessary. Use of the pre-procedural and intraprocedural imaging should avoid puncture injury to the larger arteries and portal or hepatic vein branches in the liver.

\section{Pneumothorax}

A puncture injury of the visceral pleura or lung tissue might cause pneumothorax. A small pneumothorax should be treated conservatively. For a medium or large pneumothorax, puncture aspiration or closed drainage of the thoracic cavity should be performed.

\section{Pleural Effusion}

Tumour adjacent to the diaphragm, heat energy, and tumour necrotic tissue causing pleural stimulation are common reasons for pleural effusion. A small pleural effusion should be treated conservatively. For medium and large pleural effusions, puncture aspiration or catheter drainage should be performed.

\section{Bile Duct or Gallbladder Injury}

Mild bile duct dilatation requires no therapy. Catheter drainage or cholangioplasty should be performed for moderate-to-severe obstructive jaundice. If the tumour is adjacent to the larger bile duct or gallbladder, the ablation power should be adjusted based on the pre-procedural and intraprocedural imaging and planned temperature fields.

\section{Gastrointestinal Tract Injury}

Ablation of lesions adjacent to the gastrointestinal tract can easily cause gastrointestinal injury. Gastrointestinal perforation requires gastrointestinal decompression, fasting from solids and liquids, or timely surgery, as appropriate. Pre-procedural planning, precise intraprocedural positioning, use of reasonable ablation parameter settings, and attention to the protection of the gastrointestinal tract should all precede the ablation procedure.

\section{Pericardial Tamponade}

Puncture injury of the pericardium could cause pericardial effusion leading to cardiac tamponade. A small pericardial effusion $(<100 \mathrm{~mL})$ should be closely observed. Emergency pericardial drainage should be performed if a small effusion increases or if the initial effusion is moderate or high (>100 mL).

\section{Liver Failure}

Excessive ablation volume in a single procedure, infection, massive hemorrhage, or injury to the great portal vein or bile duct might cause liver failure. A highly detailed individualized ablation plan should therefore be completed before the ablation. During the procedure, the range of ablation to each lesion should be controlled, with avoidance of injury to the larger bile duct and arteries. After the procedure, the patient's condition should be closely monitored, with active protection of liver function, supportive care, and timely treatment of complications, as required. 


\section{Skin Scald}

Skin scald can occur at the locations of the negative plate and the puncture point. For mild scalding, clean the skin and keep it dry to prevent infection. Apply scald ointment locally. Treat moderate scalding in accordance with its severity, and undertake debridement and skin grafting if necessary.

\section{Frostbite}

Cryoablation at the puncture point can cause frostbite. During the ablation process, a hot compress or a cotton ball soaked with alcohol can be applied. Frostbite can be treated by changing the wound dressing as needed to keep the skin clean and dry and to prevent infection.

\section{Cold Shock}

Cold shock is rare complication in which patients feel cold and experience a drop in blood pressure, a quickening heart rate, sweating, and other serious clinical manifestations. During the cryoablation part of the multimode procedure, the patient should be kept warm, and a hot compress with hot water bag should be applied locally. If cold shock recurs, ablation should immediately be ceased. Dopamine, fluid infusion, and other intensive care measures should be promptly undertaken. The patient should be sent to the intensive care unit if necessary.

\section{Other}

Intercostal artery injury, intercostal nerve injury, and fistulae (hepatic artery to portal vein or to hepatic vein, and bile duct to bronchia) are rare complications ${ }^{29}$.

\section{Follow-Up and Evaluation}

\section{Follow-Up}

Contrast-enhanced ст or MRI of liver and tumour marker assessment are recommended every month for the first 3 months after the ablation. If the results are negative, those examinations should be repeated every 3 months thereafter.

If the imaging examination during the follow-up period shows residual tumour, local tumour progression, or a new tumour suitable for repeat ablation, the ablation procedure can be repeated with subsequent follow-up as already described. If repeat ablation is contraindicated, other comprehensive treatment should be adopted.

Evaluation of cure considers these four aspects of the procedure ${ }^{1,2}$ :

\section{Technical success}

The target tumour has been completely ablated per the pre-procedural planning, as evaluated by intraprocedural and immediate post-procedural contrastenhanced ultrasonography, CT, or MRI.

\section{Technical efficacy}

Contrast-enhanced cт or MRI and other imaging examinations should be performed immediately and 1 month after ablation to confirm that the tumour has been completely ablated. Subsequently, imaging and tumour markers are assessed every 3 months to detect local recurrence, new lesions, or extrahepatic metastases.

\section{Complications}

Ablation-related complications are classified into short- and long-term complications per the Society of Interventional Radiology criteria. The short-term complications include problems encountered within the periprocedural period ( 24 hours to 30 days) that can be related in any way to the procedure. Long-term complications are those identified at delayed follow-up imaging that are judged to be highly likely to be a result of the ablation therapy (stricture of the biliary duct, tumour seeding along the needle track, and so on) ${ }^{1}$.

\section{Outcomes}

Local efficacy (imaging evaluation), systemic efficacy (that is, pain, relief of symptoms, and so on), change in tumour markers, quality of life, time to disease progression (or progression-free survival), and overall survival duration are evaluated during follow-up.

\section{Local Efficacy}

Contrast-enhanced ultrasonography and CT or MRI are the imaging methods commonly used to evaluate local efficacy. Integrated positron-emission tomography-cT can also be used if necessary. The response is determined by comparing the lesion at baseline with the result 1 month after ablation. According to the Modified Response Evaluation Criteria in Solid Tumors, the curative effect of ablation can be assessed as follows ${ }^{1,2,29}$ :

\section{Complete ablation}

Adequate ablation and an absence of viable tissue in the target lesion and surrounding ablation margin $(0.5-1.0 \mathrm{~cm})$ in imaging follow-up.

\section{Partial ablation or residual tumour}

Demonstration of residual viable tumour at the ablative margin in the initial follow-up imaging is referred to as "residual tumour" or "partial ablation."

\section{Local tumour progression}

"Local tumour progression" describes the appearance of tumour foci at the edge of the ablation zone after at least 1 contrast-enhanced follow-up study has shown complete ablation.

\section{New tumour}

Visible foci in the liver parenchyma outside the original ablation area on any post-procedural imaging during the follow-up period are classified as new tumours.

\section{General Evaluation}

The general evaluation includes relief of clinical symptoms and improvement in quality of life. The Quality of Life Scale, combined with a pain score (numeric rating scale) and the Eastern Cooperative Oncology Group performance status, constitute a comprehensive evaluation.

\section{Evaluation of Tumour Markers}

Ongoing examination of tumour markers such as alphafetoprotein, carbohydrate antigen 19-9, and carcinoembryonic antigen can assist in evaluating the curative 
effects of ablation and signalling tumour recurrence or progression.

\section{Clinical Outcome Evaluation}

Overall survival is the most important indicator for clinical outcome, which should be calculated from the day of ablation treatment to the day of death. During follow-up, the interval between treatment initiation and disease progression (time to progression and time to local progression) and progression-free survival or local progression-free survival should be evaluated using imaging ${ }^{30}$.

\section{SUMMARY}

Image-guided multimode ablation of solid tumours of the liver precisely conducted under imaging guidance for the treatment of hepatic malignant tumours, combines the widely used cryoablation and RFA techniques throughintelligent navigation technology. The present guideline establishes a standardized technique that requires development of an individualized treatment plan according to the patient's condition. The resulting procedure constitutes a reliable clinical solution for a minimally invasive ablation of malignant hepatic tumours to achieve symptom and quality-of-life improvements, tumour control, treatment efficiency, and prolonged survival duration.

\section{ACKNOWLEDGMENTS}

This study was supported by the National Key Research and Development Program of China: Pilot Project of Digital Diagnostic and Therapeutic Equipment Research-Clinical solutions of image-guided multimode ablation for the treatment of solid tumor (no. 2016YFC0106203).

\section{CONFLICT OF INTEREST DISCLOSURES}

We have read and understood Current Oncology's policy on disclosing conflicts of interest, and we declare that we have none.

\section{AUTHOR AFFILIATIONS}

*Department of Interventional Oncology, Fudan University Shanghai Cancer Center, ${ }^{\dagger}$ Department of Oncology, Affiliated Shanghai 6th People's Hospital, Shanghai Jiaotong University, ${ }^{\ddagger}$ Department of Interventional Radiology, Zhongshan Hospital, Fudan University, ${ }^{\S}$ Department of Interventional Radiology, Ruijin Hospital, Shanghai Jiaotong University School of Medicine, and "Department of Interventional Radiology, Changhai Hospital, Naval Medical University, Shanghai, P.R.C.

\section{REFERENCES}

1. Ahmed M, Solbiati L, Brace CL, et al. on behalf of the International Working Group on Image-Guided Tumor Ablation, the Interventional Oncology Sans Frontières Expert Panel, the Technology Assessment Committee of the Society of Interventional Radiology, and the Standard of Practice Committee of the Cardiovascular and Interventional Radiological Society of Europe. Image-guided tumor ablation: standardization of terminology and reporting criteria-a 10-year update. $J$ Vasc Interv Radiol 2014;25:1691-705.

2. Chinese Society of Liver Cancer, Chinese Society of Clinical Oncology, Liver Cancer Group, Chinese Society of Hepatology. Chinese expert consensus statement: guidelines for radiofrequency ablation therapy of liver cancer.JClin Hepatol 2011;27:236-8,244-5.

3. Breen DJ, Lencioni R. Image-guided ablation of primary liver and renal tumours. Nat Rev Clin Oncol 2015;12:175-86.
4. Narayanan G, Hosein PJ, Beulaygue IC, et al. Percutaneous image-guided irreversible electroporation for the treatment of unresectable, locally advanced pancreatic adenocarcinoma. J Vasc Interv Radiol 2017;28:342-8.

5. Yang Y. Cancer immunotherapy: harnessing the immune system to battle cancer. J Clin Invest 2015;125:3335-7.

6. Lo SS, Moffatt-Bruce SD, Dawson LA, et al. The role of local therapy in the management of lung and liver oligometastases. Nat Rev Clin Oncol 2011;8:405-16.

7. Chu KF, Dupuy DE. Thermal ablation of tumours: biological mechanisms and advances in therapy. Nat Rev Cancer 2014;14:199-208.

8. Takaki H, Cornelis F, Kako Y, et al. Thermal ablation and immunomodulation: from preclinical experiments to clinical trials. Diagn Interv Imaging 2017;98:651-9.

9. Silvestrini MT, Ingham ES, Mahakian LM, et al. Priming is key to effective incorporation of image-guided thermal ablation into immunotherapy protocols. JCI Insight 2017;2:e90521.

10. Shen Y, Liu P, Zhang A, Xu LX. Study on tumor microvasculature damage induced by alternate cooling and heating. Ann Biomed Eng 2008;36:1409-19.

11. Zhu J, Zhang Y, Zhang A, He K, Liu P, Xu LX. Cryo-thermal therapy elicits potent antitumour immunity by inducing extracellular Hsp70-dependent MDsc differentiation. Sci Rep 2016;6:27136.

12. He K, Liu P, Xu LX. The cryo-thermal therapy eradicated melanoma in mice by eliciting $\mathrm{CD} 4^{+} \mathrm{T}$-cell-mediated antitumour memory immune response. Cell Death Dis 2017;8:e2703.

13. Xue T, Liu P, Zhou Y, et al. Interleukin-6 induced "acute" phenotypic microenvironment promotes Thl antitumour immunity in cryo-thermal therapy revealed by shotgun and parallel reaction monitoring proteomics. Theranostics 2016;6:773-94.

14. Yashiro H, Nakatsuka S, Inoue M, et al. Factors affecting local progression after percutaneous cryoablation of lung tumors. J Vasc Interv Radiol 2013;24:813-21.

15. Littrup PJ, Aoun HD, Adam B, Krycia M, Prus M, Shields A. Percutaneous cryoablation of hepatic tumors: longterm experience of a large U.S. series. Abdom Radiol (NY) 2016;41:767-80.

16. Livraghi T, Solbiati L, Meloni MF, Gazelle GS, Halpern EF, Goldberg SN. Treatment of focal liver tumors with percutaneous radio-frequency ablation: complications encountered in a multicenter study. Radiology 2003;226:441-51.

17. Dong J, Liu P, Xu LX. Immunologic response induced by synergistic effect of alternating cooling and heating of breast cancer. Int J Hyperthermia 2009;25:25-33.

18. Liu K, He K, Xue T, Liu P, Xu LX. The cryo-thermal therapyinducedIL-6-richacutepro-inflammatory responsepromoted DCs phenotypic maturation as the prerequisite to $\mathrm{CD} 4^{+} \mathrm{T}$ cell differentiation. Int J Hyperthermia 2018;34:261-72.

19. Liu W, Guo H, Du X, et al. Cortical vessel imaging and visualization for image guided depth electrode insertion. Comput Med Imaging Graph 2013;37:123-30.

20. Bai JF, Liu P, Xu LX. Recent advances in thermal treatment techniques and thermally induced immune responses against cancer. IEEE Trans Biomed Eng 2014;61:1497-505.

21. Abi-Jaoudeh N, Glossop N, Dake M, et al. Electromagnetic navigation for thoracic aortic stent-graft deployment: a pilot study in swine. J Vasc Interv Radiol 2010;21:888-95.

22. Chinese Society of Liver Cancer, Chinese Anti-Cancer Association; Chinese Society of Clinical Oncology, Chinese Anti-Cancer Association; Liver Cancer Study Group, Chinese Society of Hepatology, Chinese Medical Association. Expert consensus on the norms of local ablation therapy for hepatocellular carcinoma [Chinese]. Zhonghua Gan Zang Bing Za Zhi 2011;19:257-9. 
23. Expert Committee of the National Industrial Technology Innovation Strategic Alliance for Minimally Invasive Treatment of Tumor; Expert Guidance Committee for Ablation Therapy, Society of Interventional Physicians, Chinese Medical Doctor Association; Society of Interventional Physicians, Beijing Medical Doctor Association. Expert consensus on standardized clinical application of image-guided thermal ablation of liver tumor. J Clin Hepatol 2017;33:1864-9.

24. Interventional Radiology Group, Chinese Society of Radiology. Expert consensus on the application of percutaneous radiofrequency ablation of liver cancer [Chinese]. Zhonghua Fang She Xue Za Zhi 2012;46:581-5.

25. Society of Interventional Therapy, Chinese Anti-Cancer Association; Solid-Tumor Focused Clinic, Shanghai Anti-Cancer Association. Expert consensus on the imaging-guided multimode tumor ablation therapy system for malignant hepatic tumors [Chinese]. Jie Ru Fang She Xue Za Zhi 2016;27:603-7.
26. Patel IJ, Davidson JC, Nikolic B, et al. on behalf of the Standards of Practice Committee, Cardiovascular and Interventional Radiological Society of Europe. Consensus guidelines for periprocedural management of coagulation status and hemostasis risk in percutaneous image-guided interventions. J Vasc Interv Radiol 2012;23:727-36.

27. Skonieczki BD, Wells C, Wasser EJ, Dupuy DE. Radiofrequency and microwave tumor ablation in patients with implanted cardiac devices: is it safe? Eur J Radiol 2011;79:343-6.

28. Eakin JL, Bader AM. ASA physical status classification system: is it consistentamongst providers and useful in determining need for pre-operative evaluation resources? J Clin Anesth 2017;39:73-4.

29. YeX, Fan W, Wang H, et al. Expert consensus workshop report: guidelines for thermal ablation of primary and metastatic lung tumors (2018 edition). JCancer Res Ther 2018;14:730-44.

30. Liang P, Yu J, Yu XL, et al. Percutaneous cooled-tip microwave ablation under ultrasound guidance for primary liver cancer: a multicentre analysis of 1363 treatment-naïve lesions in 1007 patients in China. Gut 2012;61:1100-1. 